\title{
Reducing Hamiltonian path to a vertex label
}

\author{
Thinh D. Nguyen
}

\section{Introduction and notations}

Given a graph $\boldsymbol{G}=(\boldsymbol{V}, \boldsymbol{E})$, we want to label all the vertices $v \in \boldsymbol{V}$ with values from $\{1,2, \ldots, n\}$ where $|\boldsymbol{V}|=n$ such that for all edges $(x, y) \in \boldsymbol{E}$ such that $|\operatorname{label}(x)-\operatorname{label}(y)| \geq$ $2 \mid$. We have to determine whether such a labelling is possible for a graph.

Now we are going to prove that doing so is an NP-complete problem. While showing that it belongs to NP is straightforward, to prove the hardness of this problem would require a reduction from a known NP-complete problem. We choose to reduce from Hamiltonian path as shown in the next section.

\section{Details of the proof}

Sketch of proof: Given a graph $\boldsymbol{G}$, consider its complement $\boldsymbol{G}^{\prime}$. The graph $\boldsymbol{G}^{\prime}$ is a YES instance of our problem if its vertices can be ordered so that adjacent vertices are not connected by an edge in $\boldsymbol{G}^{\prime}$. In other words, $\boldsymbol{G}^{\prime}$ is a YES instance of our problem if the vertices of $\boldsymbol{G}$ can be ordered so that adjacent vertices are connected. So $\boldsymbol{G}^{\prime}$ is a YES instance of our problem if and only if $\boldsymbol{G}$ has a Hamiltonian path.

Proof: Indeed, for one direction, if $\boldsymbol{G}$ is semi-Hamiltonian, then given one Hamiltonian path of it, we easily label vertices of our $G^{\prime}$. Just starting with label 1 for the starting vertex of the path, increment along the way until the ending vertex with label $n$. Intuitively, each vertex with label $i$ in $G^{\prime}$ is connected with both $i-1$ and $i+1$. So in $G^{\prime}$, the complement of $\boldsymbol{G}$, every edge $(x, y)$ must satisfy the required condition.

For the other direction, if we can label $\boldsymbol{G}^{\prime}$, we would now like to have $\boldsymbol{G}$ semi-Hamiltonian. So, we start from the vertex with label 1 , and this is our current vertex in the algorithm constructing a Hamiltonian path for $\boldsymbol{G}$. As our vertex labelling is feasible, by connectedness of $\boldsymbol{G}$, the only possible adjacent vertex of our current vertex would be a vertex with label 2 . For if this is not the case, infeasibility is the property of our vertex labelling for $\boldsymbol{G}^{\prime}$. So, our current vertex now has

label 2. Repeat this procedure until we reach the vertex with label $n$. Clearly, we obtain a Hamiltonian path for $\boldsymbol{G}$.

\section{References}

[1] Thomas H. Cormen, Charles E. Leiserson, Ronald L. Rivest, and Clifford Stein, Introduction to Algorithms 